\title{
Análise química em órgãos de reserva de algumas herbáceas e arbóreas ocorrentes na flora do Espírito Santo, Brasil
}

\author{
Joscineia Kelli Clippel ${ }^{1}$, Hallan Nunes Chamon do Carmo ${ }^{2}$, Luis Cláudio Zanette Nascimento² e \\ Geraldo Rogério Faustini Cuzzuol ${ }^{2,3}$
}

Recebido em 13/07/2006. Aceito em 11/03/2008

\begin{abstract}
RESUMO - (Análise química em órgãos de reserva de algumas herbáceas e arbóreas ocorrentes na flora do Espírito Santo, Brasil). Foi investigada a composição de carboidratos não estruturais em órgãos subterrâneos de reserva das herbáceas Dioscorea sp. 1 e Dioscorea sp. 2 (Dioscoreaceae), Hedychium coronarium J. König (Zingiberaceae), Hippeastrum reticulatum (L’Hérit.) Herb. (Amaryllidaceae), Scadoxus multiflorus (Martyn) Raf. (Amaryllidaceae), Prescottia nivalis Barb. Rodr. (Orchidaceae) e Sinningia aghensis Chautems (Gesneriaceae). O trabalho objetivou, também, avaliar os teores dos polissacarídeos de reserva de parede celular (PRPC) e de nutrientes minerais em sementes de Cassia fistula L., Cassia grandis L.f., Canavalia rosea (Sw.) DC., Erythrina variegata L., Hymenaea courbaril L. e Ormosia arborea (Vell.) Harms pertencentes à família Leguminosae. A espécie que apresentou maior conteúdo de frutose foi a bulbosa H. reticulatum (25\% MS) sugerindo a presença de frutanos em seus órgãos subterrâneos. Os maiores teores de carboidratos solúveis totais, especialmente a sacarose (63\% MS), foram observados em rizomas de P. nivalis, indicando que esse carboidrato desempenhe papel osmorregulador nessa rupestre revivescente. Quanto aos polissacarídeos de reserva de parede celular, maiores valores foram encontrados em sementes de H. courbaril (70\% MS), C. grandis (53\% MS) e C. rosea (40\% de MS). Valores intermediários foram obtidos em C. fistula (24\% de MS) e E. variegata (11\% MS). Sementes de O. arborea mostraram os mais baixos teores de PRPC não ultrapassando ao valor de $3 \%$ da MS. Trabalhos anteriores denotam que o polissacarídeo de reserva de $H$. courbaril é do tipo xiloglucano, enquanto o de $C$. grandis, C. fistula e C. rosea seja do tipo galactomanano. A identificação do tipo de polissacarídeo de E. variegata e de $O$. arborea depende de análises futuras através da cromatografia líquida de alta eficiência. Dos nutrientes minerais, sementes de C. rosea e E. variegata destacaram pelos elevados teores de $\mathrm{N}\left(60 \mathrm{~g}_{\mathrm{kg}} \mathrm{kg}^{-1}\right)$ entre os macronutrientes e de Fe $\left(160-170 \mathrm{mg} \cdot \mathrm{g}^{-1}\right)$ e Zn $\left(56-87 \mathrm{mg} \cdot \mathrm{g}^{-1}\right)$ dentre os micronutrientes. Como constatado no presente trabalho, os estudos relacionados à bioprospecção revelam resultados promissores mostrando o grande potencial tecnológico de sementes e de órgãos subterrâneos de armazenamento encontrados na flora do Espírito Santo.
\end{abstract}

Palavras-chave: carboidratos não estruturais, bulbos, polissacarídeos, sementes, tubérculos

\begin{abstract}
Chemical analysis of storage organs in herb and tree species from Espírito Santo State, Brazil). The composition of non-structural carbohydrates was investigated in the underground organs of the herbs Dioscorea sp. 1 and Dioscorea sp. 2 (Dioscoreaceae); Hedychium coronarium J. König (Zingiberaceae); Hippeastrum reticulatum (L'Hérit.) Herb. (Amaryllidaceae); Scadoxus multiflorus (Martyn) Raf. (Amaryllidaceae), Prescottia nivalis Barb. Rodr. (Orchidaceae) and Sinningia aghensis Chautems (Gesneriaceae). We was also analyzed the levels of cell-wall storage polysaccharides (CWSP) and mineral nutrients in seeds of Cassia fistula L., Cassia grandis L.f., Canavalia rosea (Sw.) DC., Erythrina variegata L., Hymenaea courbaril L. and Ormosia arborea (Vell.) Harms from the Leguminosae family. H. reticulatum showed the highest fructose levels (25\% dry mass) suggesting the presence of fructans in the underground organs. The highest levels of total soluble carbohydrates, especially sucrose (63\%), were observed in rhizomes of P. nivalis indicating that this carbohydrate may act as an osmoregulator in this rock-dwelling resurrection plant. In relation to CWSP, high values were found in H. courbaril (70\%), C. grandis (53\%) and C. rosea (40\%) seeds. Intermediate values were found in C. fistula (24\%) and E. variegata $(11 \%)$. O. arborea seeds showed the lowest amount of $\mathrm{WCP}(<3 \%$ dry mass). Previous work has shown that the storage polysaccharides of $H$. courbaril are of the xyloglucan type while in C. grandis, C. fistula and C. rosea they are of the galactomanan type. Identification of the polysaccharide in E. variegata and $O$. arborea depends on future analyses by HPLC. In relation to mineral nutrients, $C$. rosea and E. variegata seeds stood out due to higher concentrations of $\mathrm{N}\left(60 \mathrm{~g} \cdot \mathrm{kg}^{-1}\right)$ among the macronutrients and higher concentrations of Fe (160-170 mg. $\left.\mathrm{g}^{-1}\right)$ and $\mathrm{Zn}\left(56-87 \mathrm{mg} \cdot \mathrm{g}^{-1}\right)$ among the micronutrients. As shown here, bioprospecting studies reveal promising results showing the great technological potential of seeds and underground storage organs in the Espírito Santo flora.
\end{abstract}

Key words: non-structural carbohydrates, bulbs, polysaccharides, seeds, tubers

\footnotetext{
Parte da Dissertação de Mestrado do primeiro Autor

2 Universidade Federal do Espírito Santo, Pós-Graduação em Biologia Vegetal, Fernando Ferrrari 514, Campus Universitário Alaor Queiroz de Araújo, 29060-900 Vitória, ES, Brasil

3 Autor para correspondência: cuzzuol@npd.ufes.br,gcuzzuol@gmail.com
} 


\section{Introdução}

A megadiversidade em florestas tropicais fornece uma grande variedade de alimentos através de seus órgãos subterrâneos de armazenamento e de suas sementes onde é possível encontrar diversos produtos de aplicação na indústria farmacêutica e alimentícia (Gonçalves et al. 2002). Mesmo abrigando mais de 20 mil espécies de plantas, pouco se sabe sobre a composição química do compostos orgânicos das plantas de Mata Atlântica brasileira; especialmente na flora do Estado do Espírito Santo considerada uma das maiores diversidades de espécies vegetais arbóreas do planeta (Thomaz \& Monteiro 1997). Silva et al. (2005) cita que pouco mais de 448 espécies da biodiversidade brasileira são usadas como alimento, representando apenas $2 \%$ das espécies listadas.

Embora as espécies nativas brasileiras constituem um dos recursos naturais mais importantes da biosfera, só recentemente trabalhos sobre a sua utilização vêm sendo desenvolvidos. Frutos da palmeira Syagrus coronata (licuri) têm-se mostrado importante complemento vitamínico sendo indicado como fonte de alimento para a população da área rural da caatinga baiana (Crepaldi et al. 2001). As sementes de plantas tropicais são outros órgãos com grande potencial nutritivo à dieta animal (Gonçalves et al. 2002), porém pouco exploradas em trabalhos de bioprospecção. Os carboidratos, vitaminas e minerais são as reservas de maior interesse em sementes tropicais (Yexkull \& Mutert 1995). Em levantamento realizado na vegetação de cerrado e de Mata Atlântica do Estado de São Paulo na década de 80 e 90, foram identificados polissacarídeos com funções fisiológicas. Dentre esses, se destacam os polissacarídeos do tipo frutanos em tubérculos de Asteraceae e em raízes tuberosas de Amaranthaceae (Carvalho \& Figueiredo-Ribeiro 2001), além de xiloglucanos e galactomananos, importantes polissacarídeos de reserva de parede celular (PRPC) encontrados abundantemente em sementes de Leguminosae (Buckeridge et al. 2004).

Os frutanos destacam-se devido a uma série de benefícios à saúde humana que despertaram a atenção da indústria alimentícia mundial. $\mathrm{O}$ fato dos frutanos não serem absorvidos pelo organismo humano fez desses polímeros de frutose uma potente fonte alternativa de açúcar de baixa caloria ideal para uso em diabéticos. No cólon do intestino humano, os frutanos funcionam como fonte de carbono para as bifidobactérias inibidoras da proliferação das bactérias patogênicas, reduzindo dessa forma a incidência de doenças intestinais como o câncer de cólon (Coussement \& Franck 1998). A possibilidade de serem utilizados como estabilizante de alimentos, cooperou para que os frutanos fossem empregados com sucesso como espessantes de iogurtes, sorvetes e cremes.

Propriedades físico químicas especiais também são encontrados nos PRPC em sementes que têm permitido seu uso em diversos setores da indústria. Semelhante aos frutanos, os PRPC de sementes são fibras biológicas que quando utilizados na dieta humana evitam o excesso de absorção de gorduras e açúcares, controlando doenças como a hipercolesterolemia e o diabetes (Buckeridge et al. 2004).

Além das famílias Liliaceae, Asteraceae e Amaryllidaceae, outros grupos que se desenvolvem em locais que passam por algum tipo estresse ambiental como restrição hídrica e nutricional e de elevada salinidade podem acumular carboidratos do tipo frutanos (Flores \& Flores 1997; Dennis \& Blakeley 2000; DiasTagliacozzo et al. 2004; Cuzzuol et al. 2005). A ocorrência de herbáceas apresentando órgãos subterrâneos espessados das famílias Amaryllidaceae, Gesneriaceae, Zingiberaceae, Orchidaceae e Dioscoreaceae em formações rochosas, arenosas e encharcadas da vegetação do ES sugerem a presença de polissacarídeos funcionais. Outras plantas da família Leguminosae como a herbácea Canavalia rosea (Sw.) DC ocorrente na formação psamófila-reptante de restinga (Thomaz \& Monteiro 1993) e a arbórea Hymenea courbaril L. nas florestas do Espírito Santo (Thomaz \& Monteiro 1997) deve possibilitar a presença de elevados teores de PRPC em suas sementes. Essas observações determinaram na realização desse trabalho que teve como objetivo a caracterização dos carboidratos não-estruturais e dos nutrientes minerais em algumas espécies dos grupos taxonômicos citados anteriormente.

\section{Material e métodos}

Material vegetal - Esse estudo foi realizado no período de março/2003 a fevereiro/2004 no laboratório de Ecofisiologia Vegetal do Setor de Botânica do Departamento de Ciências Biológicas da Universidade Federal do Espírito Santo (UFES). Foram analisados os carboidratos dos órgãos subterrâneos espessados de algumas herbáceas (Fig. 1) e de sementes em algumas arbóreas ocorrentes na vegetação do Espírito Santo identificadas, previamente, na fase floral no herbário de Vitória localizado na Universidade Federal do Espírito Santo (UFES). As espécies herbáceas analisadas foram Dioscorea sp. 1 e Dioscorea sp. 2 (Dioscoreaceae); Hedychium coronarium J. König (Zingiberaceae) originária do Himalaia; Hippeastrum reticulatum (L'Hérit.) Herb. (Amaryllidaceae); Prescottia nivalis Barb. Rodr. (Orchidaceae); Scadoxus multiflorus (Martyn) Raf. (Amaryllidaceae) proveniente da África e Sinningia 
aghensis Chautems (Gesneriaceae). As plantas dos gêneros Dioscorea, Hedychium e Scadoxus foram coletadas na reserva Biológica de Duas Bocas, município de Caricacica (20 $\left.25^{\prime} 50^{\prime \prime} \mathrm{S}, 40^{\circ} 25^{\prime} 12^{\prime \prime} \mathrm{W}\right)$ enquanto as rupestres Prescottia nivalis e Sinningia aghensis foram coletadas no Morro do Cruzeiro, município de Vila Velha (20²0'12''S, 40¹7'28''W). As plantas de Hippeastrum foram coletadas na formação de Mata Seca da restinga do Parque Estadual Paulo César Vinha localizado em

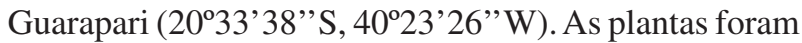

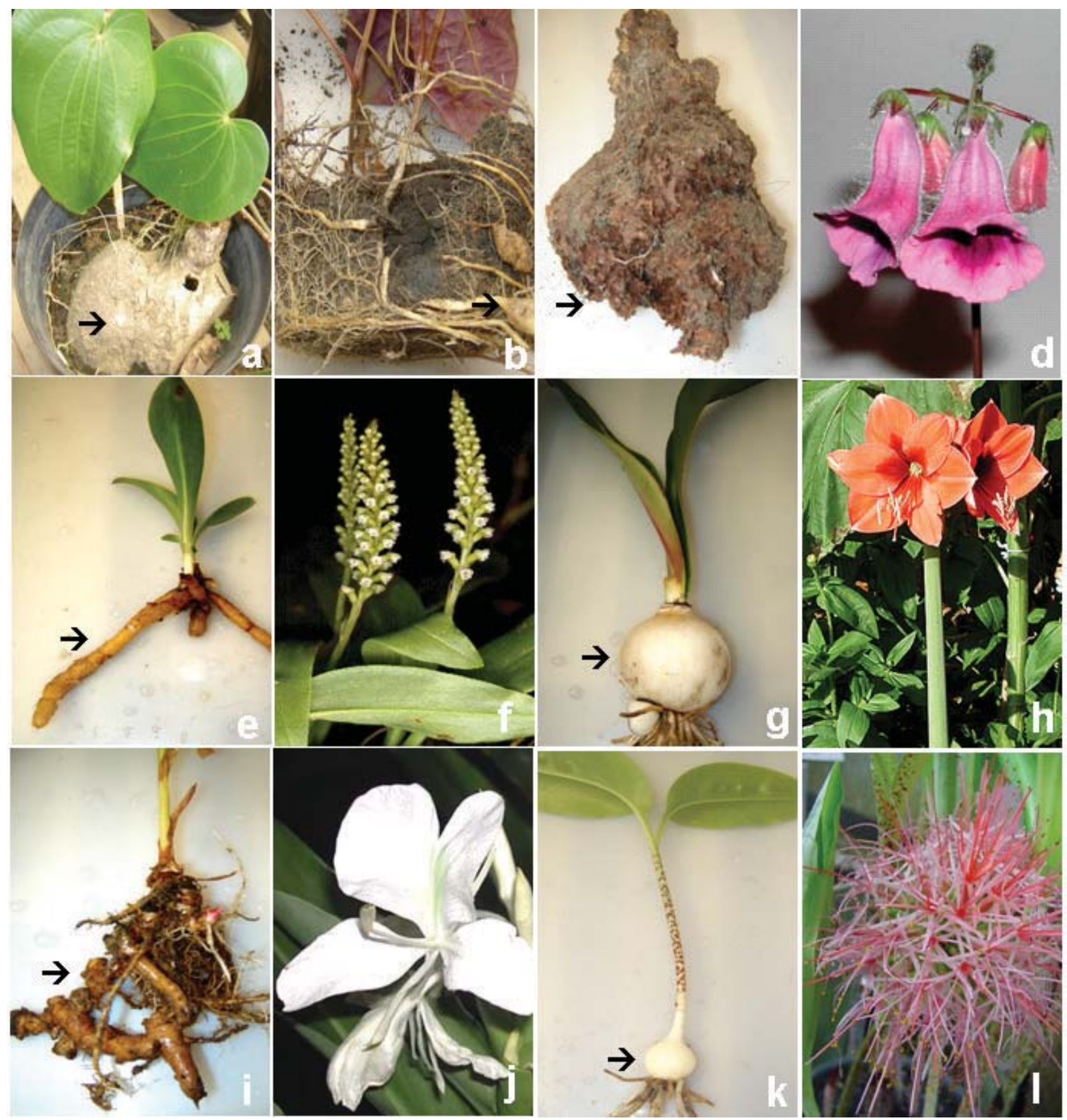

Figura 1. Aspectos morfológicos de (a) tubérculos de Dioscorea sp. 1 (Dioscoreaceae); (b) tubérculos de Dioscorea sp. 2 (Dioscoreaceae); (c) tubérculos de Sinningia aghensis Chautems (Gesneriaceae); (d) inflorescência de S. aghensis (foto de Peter Shalit); (e) rizomas de Prescottia nivalis Barb. Rodr (Orchidaceae); (f) inflorescências de P. nivalis (foto de Marcos A. Campacci); (g) bulbos de Hippeastrum reticulatum Herb. (Amaryllidaceae); (h) flor de H. reticulatum foto de G. D. Carr ; (i) rizomas de Hedychium coronarium J. Koening (Zingiberaceae); (j) flor de H. coronarium (foto de G.D. Carr); (k) bulbos de Scadoxus multiflorus (Martyn) Rafyn. (Amaryllidaceae); (l) flor de S.multiflorus (foto de P. Bambs). As setas indicam os órgãos subterrâneos analisados. 
envasadas em terra e acondicionadas em casa de vegetação do Departamento de Ciências Biológicas da UFES para posterior extração dos carboidratos. Todas as plantas se encontravam em estádio vegetativo.

Para a análise dos polissacarídeos de reserva de parede celular, foram utilizadas sementes de Cassia fistula L., Cassia grandis L.f., Canavalia rosea (Sw.) DC., Erythrina variegata L., Hymenaea courbaril L. e Ormosia arborea (Well.) Harms, todas da família Leguminosae coletadas no período de outubro a dezembro/2004. Sementes de H. courbaril foram coletadas na Reserva Biológica de Santa Lúcia situada no município de Santa Teresa-ES (1956'10's, 40³6'06''W). C. fistula, C. grandis e E. variegata foram coletadas no Campus Universitário Alaor Queiroz de Araújo da UFES em Vitória-ES (20¹9'09' S, $\left.40^{\circ} 20^{\prime} 50^{\prime \prime} \mathrm{W}\right)$, e $O$. arborea em fragmentos florestais no município de Domingos Martins (20²1'49' S, $40^{\circ} 39^{\prime} 35^{\prime}$ 'W). Sementes de C. rosea foram coletadas na restinga do Parque Estadual Paulo César Vinha, localizado em Guarapari (20³3'38'S, 40²3'26"W).

Extração e quantificação dos carboidratos em órgãos subterrâneos - A metodologia empregada para extração dos carboidratos foi a de Carvalho et al. (1998) originalmente proposta por Pollock \& Jones (1979) com algumas modificações. Fragmentos de $1 \mathrm{~g}$ de MF dos órgãos subterrâneos foram fervidos em etanol $80 \%$ durante 5 minutos, homogeneizados e submetido à extração dos oligossacarídeos em banho-maria $80{ }^{\circ} \mathrm{C}$ por 20 minutos. $\mathrm{O}$ extrato foi centrifugado a $3.000 \mathrm{~g}$ por 15 minutos e separado o sobrenadante (fração etanólica). Essa operação foi repetida mais duas vezes. O resíduo foi ressuspendido em $15 \mathrm{~mL}$ de água destilada, aquecido em banho-maria a $60{ }^{\circ} \mathrm{C}$ por 30 minutos e o homogeneizado filtrado a vácuo, em tecido de algodão. Essa operação foi repetida mais uma vez. Os resíduos sólidos foram reservados em $-10^{\circ} \mathrm{C}$ para posterior análise do amido. As frações etanólicas e aquosas foram concentradas no rotoevaporador e armazenadas em freezer. Para a determinação da massa seca dos órgãos subterrâneos por unidade de massa fresca, amostras de $1 \mathrm{~g}$ de massa fresca dos órgãos subterrâneos $(\mathrm{n}=6)$ foram mantidas em estufa $65^{\circ} \mathrm{C}$ por 3 dias.

Determinação dos carboidratos - Foi utilizado o método do fenol-sulfúrico (Dubois et al. 1956) para determinação da concentração dos carboidratos solúveis totais e a leitura realizada em espectrofotômetro (Fento 490 plus) a $490 \mathrm{~nm}$ após o resfriamento. Como padrão utilizou-se alíquotas de glicose de 0 a $50 \mathrm{mg}$. Para o conteúdo da sacarose adotou-se o método descrito por Riazi et al. (1985) que consiste na fervura da amostra por 5 minutos com hidróxido de potássio $5,4 \mathrm{~N}$ para a degradação dos carboidratos redutores e leitura realizada a $620 \mathrm{~nm}$. O conteúdo de frutose na forma livre e combinada presente nos extratos foi estimado pela reação de hidrólise ácida em antrona em banho-maria $37^{\circ} \mathrm{C}$ por 45 minutos (Jermyn 1956). Utilizou-se frutose como padrão com alíquotas de 0 a $50 \mu \mathrm{g}$ e leitura em espectrofotômetro a $620 \mathrm{~nm}$. O conteúdo de glicose na forma livre presente nos extratos foi estimado através do método enzimático (kit da BioSystem) em incubação à $25^{\circ} \mathrm{C}$ por 5 min e leitura em $500 \mathrm{~nm}$. A extração do amido foi realizada em ácido perclórico $52 \%$ segundo McCready et al. (1950) e a quantificação do amido pelo equivalente de glicose pela reação da antrona (Jermyn 1956) e a leitura realizada em espectrofotômetro, a $620 \mathrm{~nm}$.

Extração e quantificação dos polissacarídeos de sementes - As sementes de cada espécie foram desidratadas em estufa $70{ }^{\circ} \mathrm{C}$ durante 5 dias quando alcançou a massa seca constante. As sementes com testas mais rígidas foram submetidas a escarificação mecânica e embebidas por 48 horas. Após a remoção da testa, foi feita a trituração das sementes em liquidificador e o pó transferido para estufa com temperatura média de $70{ }^{\circ} \mathrm{C}$, onde permaneceu por um período de três dias quando alcançou a massa seca constante. O material foi armazenado em frascos de vidro, em temperatura ambiente. Devido às diferenças de tamanho das sementes entre as espécies estudadas, utilizou-se $5 \mathrm{~g}$ de massa seca triturada para a extração dos polissacarídeos de reserva de parede celular de sementes seguindo a metodologia de Saeman et al. (1945). As amostras foram colocadas em um erlenmeyer contendo $100 \mathrm{~mL}$ de água destilada, sobre uma placa aquecedora a $80{ }^{\circ} \mathrm{C}$, permanecendo nessas condições por 4 horas, em agitação contínua. Transcorrido esse tempo, o extrato foi filtrado em tecido algodão, e o filtrado foi transferido para novo erlenmeyer acrescido de $300 \mathrm{~mL}$ de álcool etílico comercial, na proporção de 1:3 v/v. Para precipitação dos polissacarídeos a mistura ficou em repouso por 24 horas em $5^{\circ} \mathrm{C}$. O precipitado foi lavado com acetona, seco em estufa a $60^{\circ} \mathrm{C}$ e dissolvido em água destilada com agitação contínua. O homogeneizado foi concentrado em rotaevaporador rotatório até completa secagem. A massa do polissacarídeo foi determinada pela diferença da massa do balão volumétrico vazio e após a secagem do precipitado. Para determinar o grau de pureza dos polissacarídeos extraídos, foram quantificados os carboidratos solúveis totais dos extratos pelo método do fenol-sulfúrico como descrito previamente usando-se D-glicose como padrão na concentração de 0 a $50 \mu \mathrm{g}$. Os tubos foram agitados para homogeneização da mistura, esfriados, seguindo-se as leituras em espectrofotômetro a $490 \mathrm{~nm}$. 
Determinação dos nutrientes minerais de sementes Análises de macronutrientes e micronutrientes minerais das sementes secas trituradas foram realizadas pelo Laboratório de Análise Química de Solos e Plantas do Instituto Capixaba de Pesquisa e Extensão Rural (INCAPER) da Estação Venda Nova do Imigrante, ES. Os métodos utilizados para essas análises estão pormenorizadamente descritos em Bataglia et al. (1983) e EMBRAPA (1997).

\section{Resultados}

Na Fig. 2 são apresentadas as concentrações dos carboidratos solúveis totais (CST) nas frações etanólica e aquosa. Maiores valores de CST foram encontrados em bulbos de $H$. reticulatum (945 mg.g ${ }^{-1} \mathrm{MS}$ ), rizomas de $P$. nivalis ( $837 \mathrm{mg} . \mathrm{g}^{-1} \mathrm{MS}$ ) e bulbos de $S$. multiflorus (310 mg.g ${ }^{-1} \mathrm{MS}$ ), especialmente na fração etanólica de $P$. nivalis e $S$. multiflorus e, aquosa em $H$. reticulatum. Os tubérculos de Dioscorea sp. 1, Dioscorea sp. 2 e $S$. aghensis e a rizomatosa $H$. coronarium apresentaram os mais baixos valores de CST sendo que para Dioscorea sp. 2 e $H$. coronarium maiores valores de CST foram obtidos na extração aquosa enquanto em Dioscorea sp. 1 e em $S$. aghensis, na etanólica.

Dos CST, a sacarose foi o componente em maior concentração (Fig. 3), especialmente nas espécies bulbosas $H$. reticulatum e $S$. multiflorus e na rizomatosa $P$. nivalis (660 mg.g ${ }^{-1}$ da MS). Maior concentração de sacarose foi encontrada na fração aquosa de H. reticulatum (531 mg.g ${ }^{-1}$ de $\mathrm{MS}$ ) enquanto em P. nivalis, na fração etanólica (470 mg.g $\mathrm{g}^{-1} \mathrm{de} \mathrm{MS}$ ). S. multiflorus foi a terceira espécie com maior teor desse dissacarídeo, cujo valor de 146 mg.g ${ }^{-1}$ de MS na fração etanólica foi três vezes superior ao encontrado na fração aquosa. Das plantas tuberosas Dioscorea sp.1 e

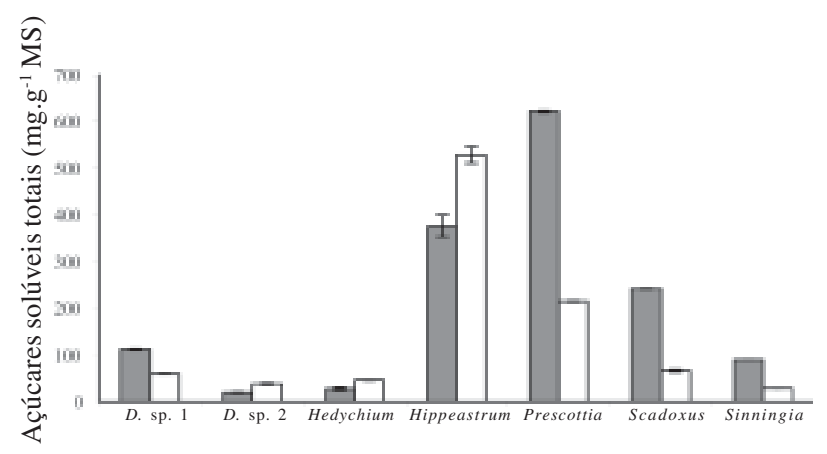

Figura 2. Concentração de açúcares totais solúveis nas frações etanólicas ( $\square$ ) e aquosas ( $\square$ ) nos órgãos subterrâneos de $D$. sp. 1 (Dioscorea sp. 1), D. sp. 2 (Dioscorea sp. 2), Hedychium coronarium J. König, Hippeastrum reticulatum (L'Hérit.) Herb., Prescottia nivalis Barb. Rodr., Scadoxus multiflorus (Martyn) Raf. e Sinningia aghensis Chautems ocorrentes na flora do Espírito Santo, Brasil. Barras representam o erro padrão $(n=6)$.
S. aghensis foram as que mostraram maiores concentrações de sacarose $\left( \pm 70 \mathrm{mg} \cdot \mathrm{g}^{-1} \mathrm{de} \mathrm{MS}\right)$ na fração aquosa enquanto valores insignificantes foram encontrados nas frações etanólicas de seus tubérculos.

A frutose livre e combinada foi o segundo componente mais representativo dos CST (Fig. 3). Mais uma vez, as espécies que apresentaram maiores concentrações de sacarose também mostraram maiores valores de frutose sendo que a concentração desse monossacarídeo foi desprezível na fração aquosa para todas as espécies investigadas. Dessa forma, os maiores valores de frutose foram obtidos entre as plantas bulbosas $H$. reticulatum (246 $\mathrm{mg} . \mathrm{g}^{-1}$ de $\mathrm{MS}$ ), seguida de S. multiflorus (85 mg. $\mathrm{g}^{-1}$ de MS) e, da rizomatosa, P. nivalis (67 mg.g $\mathrm{g}^{-1}$ de MS). As plantas de Dioscorea sp. 1, S. aghensis, H. coronarium e Dioscorea sp. 2 mostraram os mais baixos valores de frutose que variou de 25 a 47 mg.g ${ }^{-1}$ da MS.
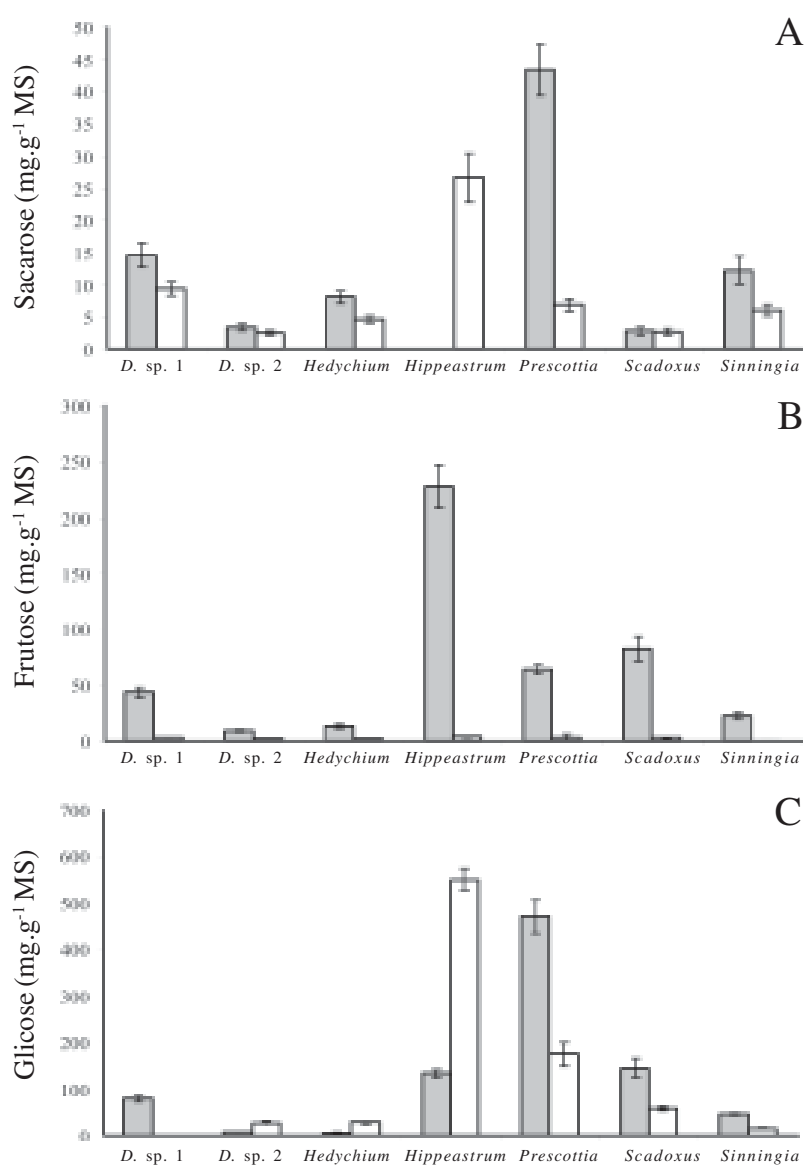

Figura 3. Concentrações de sacarose (A), frutose (B) e de glicose (C) como parte integrante dos açúcares totais solúveis nas frações etanólicas $(\square)$ e aquosas ( $\square$ ) dos órgãos subterrâneos de $D$. sp. 1 (Dioscorea sp. 1), D. sp. 2 (Dioscorea sp. 2), Hedychium coronarium J. König, Hippeastrum reticulatum (L'Hérit.) Herb., Prescottia nivalis Barb. Rodr., Scadoxus multiflorus (Martyn) Raf. e Sinningia aghensis Chautems ocorrentes na flora do Espírito Santo, Brasil. Barras representam o erro padrão $(n=6)$. 
A glicose livre foi o componente em menor concentração entre os CST variando de 6 a $50 \mathrm{mg} \cdot \mathrm{g}^{-1} \mathrm{de}$ MS (Fig. 3). Semelhante ao observado para a frutose, maiores valores de glicose foi encontrados na fração aquosa de $H$. reticulatum, representando apenas $24 \mathrm{mg} . \mathrm{g}^{-1}$ da MS, e na fração de oligossacarídeo de P. nivalis (44 mg. $\mathrm{g}^{-1}$ de MS). Das espécies estudadas, a que apresentou maior valor desse açúcar foi $P$. nivalis (50 mg. $\mathrm{g}^{-1}$ de MS) enquanto $S$. multiflorus foi a espécie que mostrou menor valor desse monossacarídeo (6 mg.g $\mathrm{g}^{-1}$ de MS).

Os resultados sobre o conteúdo de amido apresentados na Fig. 4 mostram que as plantas tuberosas da família Dioscoreaceae possuem os mais elevados teores desse polissacarídeo chegando ao valor de $520 \mathrm{mg} \cdot \mathrm{g}^{-1}$ de MS em Dioscorea sp.1 e $129 \mathrm{mg} \cdot \mathrm{g}^{-1} \mathrm{de}$ MS em Dioscorea sp.2. S. multiflorus, H. coronarium e H. reticulatum apresentaram valores intermediários (20 a $40 \mathrm{mg} . \mathrm{g}^{-1}$ de MS), enquanto $P$. nivalis e S. aghensis mostraram os valores mais baixos, inferiores a $10 \mathrm{mg} \cdot \mathrm{g}^{-1}$ de MS.

Quanto aos polissacarídeos de reserva de parede celular (PRPC) de sementes das espécies de

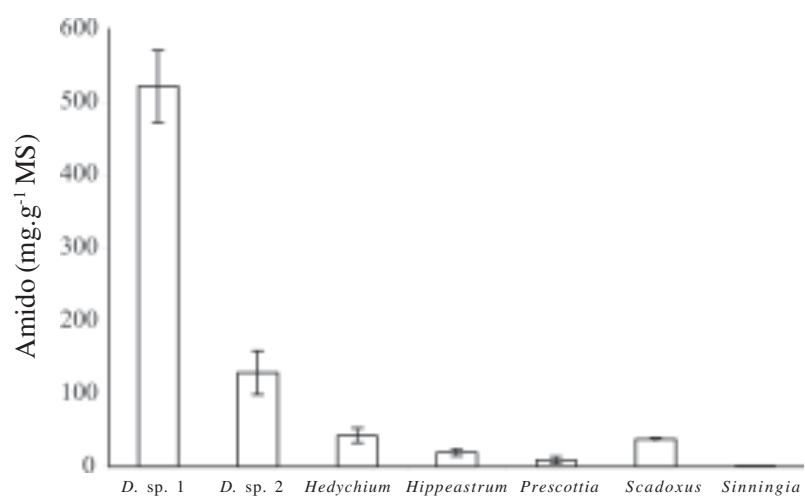

Figura 4 Concentração de amido nos órgãos subterrâneos de $D$. sp. 1 (Dioscorea sp. 1), D. sp. 2 (Dioscorea sp. 2), Hedychium coronarium J. König, Hippeastrum reticulatum (L'Hérit.) Herb., Prescottia nivalis Barb. Rodr., Scadoxus multiflorus (Martyn) Raf. e Sinningia aghensis Chautems ocorrentes na flora do Espírito Santo, Brasil. Barras representam o erro padrão $(n=3)$.
Leguminosae (Fig. 5), H. courbaril, C. grandis e C. rosea detêm maiores concentrações desses carboidratos representando 730,530 e $400 \mathrm{mg} \cdot \mathrm{g}^{-1}$ de MS, respectivamente, enquanto valores intermediários foram obtidos em $C$. fistula (240 mg.g ${ }^{-1}$ de MS) e E. variegata $\left(110 \mathrm{mg} \cdot \mathrm{g}^{-1}\right.$ de MS). Sementes de O. arborea mostraram os mais baixos teores de PRPC não ultrapassando ao valor de $30 \mathrm{mg} \cdot \mathrm{g}^{-1}$ da MS.

Quanto aos nutrientes minerais (Tab. 1), C. rosea e E. variegata foram as espécies que apresentaram maiores teores dos macronutrientes $\mathrm{N}, \mathrm{P}, \mathrm{K}, \mathrm{Ca}, \mathrm{Mg}$ e $\mathrm{S}$, e de $\mathrm{B}, \mathrm{Fe}, \mathrm{Zn}$ e $\mathrm{Cu}$ entre os micronutrientes. $O$. arborea mostrou maior teor de Mn.. De maneira geral, a proporção dos macronutrientes foi de $\mathrm{N}>\mathrm{K}>\mathrm{P}>\mathrm{Ca}$ em C. fistula, C. grandis, C. rosea e O. arborea seguido de menores teores de $\mathrm{Mg}$ e S. Proporcionalidades diferentes foram encontradas em $E$. variegata onde $\mathrm{N}>\mathrm{K}>\mathrm{Ca}>\mathrm{Mg}>\mathrm{P}>\mathrm{S}$ e em $H$. courbaril em que $\mathrm{N}>\mathrm{K}>\mathrm{P}>\mathrm{Mg}>\mathrm{Ca}>\mathrm{S}$. A proporção dos micronutrientes foi de $\mathrm{Fe}>\mathrm{Zn}>\mathrm{B}>\mathrm{Mn}>\mathrm{Cu}$ em $C$. fistula e $C$. grandis, $\mathrm{Fe}>\mathrm{Zn}>\mathrm{Mn}>\mathrm{B}>\mathrm{Cu}$ em C. rosea e E. variegata e $\mathrm{Fe}>\mathrm{Mn}>\mathrm{Zn}>\mathrm{B}>\mathrm{Cu}$ em $H$. courbaril e $O$. arborea.

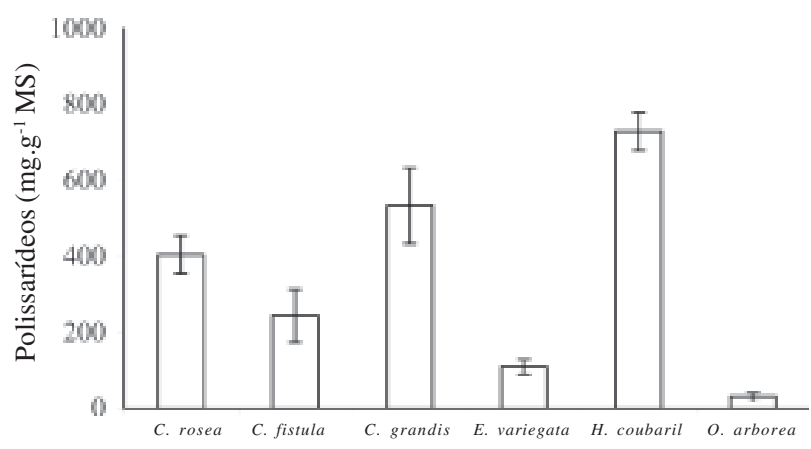

Figura 5. Concentração de polissacarídeos de reserva de parede celular (PRPC) de sementes das espécies Canavalia rosea (Sw.) DC., Cassia fistula L., Cassia grandis L.f., Erythrina variegata L., Hymenaea courbaril L. e Ormosia arborea (Vell.) Harms (Leguminosae) ocorrentes na flora do Espírito Santo, Brasil. Barras representam o erro padrão $(\mathrm{n}=3)$.

Tabela 1. Concentração de macro e micronutrientes em sementes das espécies Canavalia rosea (Sw.) DC., Cassia fistula L., Cassia grandis L.f., Erythrina variegata L., Hymenaea courbaril L. e Ormosia arborea (Vell.) Harms (Leguminosae) ocorrentes na flora do Espírito Santo, Brasil. $( \pm)$ representa o erro padrão $(\mathrm{n}=3)$.

\begin{tabular}{|c|c|c|c|c|c|c|c|c|c|c|c|}
\hline \multirow[t]{2}{*}{ Espécies } & $\mathrm{N}$ & $\mathrm{P}$ & K & $\mathrm{Ca}$ & $\mathrm{Mg}$ & S & B & $\mathrm{Fe}$ & $\mathrm{Mn}$ & $\mathrm{Zn}$ & $\mathrm{Cu}$ \\
\hline & \multicolumn{6}{|c|}{ g. $\mathrm{kg}^{-1}$} & \multicolumn{5}{|c|}{$\mathrm{mg} \cdot \mathrm{g}^{-1}$} \\
\hline C. rosea & $59 \pm 0,7$ & $3,4 \pm 0,2$ & $10 \pm 0,5$ & $2,2 \pm 0,5$ & $2,0 \pm 0,1$ & $2,0 \pm 0,2$ & $15 \pm 1,2$ & $170 \pm 31$ & $20 \pm 0,8$ & $87 \pm 5,9$ & $14 \pm 0,2$ \\
\hline C. fistula & $29 \pm 0,6$ & $2,2 \pm 0,1$ & $6,5 \pm 1,3$ & $1,9 \pm 0,1$ & $1,4 \pm 0,3$ & $0,7 \pm 0,1$ & $16 \pm 0,8$ & $77 \pm 15$ & $06 \pm 0,1$ & $18 \pm 2,0$ & $01 \pm 0,0$ \\
\hline C. grandis & $16 \pm 1,4$ & $1,5 \pm 0,3$ & $4,0 \pm 0,6$ & $0,9 \pm 0,2$ & $0,9 \pm 0,1$ & $0,4 \pm 0,1$ & $09 \pm 0,5$ & $40 \pm 4,1$ & $03 \pm 0,1$ & $12 \pm 1,5$ & $01 \pm 0,0$ \\
\hline E. variegata & $60 \pm 1,6$ & $4,1 \pm 0,1$ & $11 \pm 1,3$ & $6,4 \pm 0,3$ & $4,4 \pm 0,3$ & $1,7 \pm 0,3$ & $21 \pm 1,5$ & $160 \pm 28$ & $27 \pm 1,2$ & $56 \pm 8,1$ & $18 \pm 0,3$ \\
\hline H. courbaril & $13 \pm 0,2$ & $1,6 \pm 0,1$ & $4,5 \pm 0,8$ & $1,2 \pm 0,2$ & $1,4 \pm 0,2$ & $0,6 \pm 0,1$ & $11 \pm 0,5$ & $97 \pm 8,8$ & $36 \pm 1,1$ & $22 \pm 0,7$ & $02 \pm 0,1$ \\
\hline O. arborea & $20 \pm 0,5$ & $1,6 \pm 0,3$ & $3,5 \pm 0,5$ & $1,5 \pm 0,2$ & $1,5 \pm 0,2$ & $0,4 \pm 0,1$ & $21 \pm 0,7$ & $103 \pm 9,4$ & $80 \pm 8,2$ & $26 \pm 0,5$ & $02 \pm 0,0$ \\
\hline
\end{tabular}




\section{Discussão}

Pelo exposto, nota-se a existência de um padrão de proporcionalidade na composição dos carboidratos com a sacarose aparecendo em maior concentração, a frutose com valores intermediários seguidos de baixíssimas concentrações de glicose livre. Das plantas estudadas, bulbos de $H$. reticulatum (Amaryllidaceae) destacaramse por apresentarem maiores teores de CST (94,5\% MS) constituído, basicamente, de sacarose (66,5\% MS) e frutose (25\% MS). Comportamento similar, porém, com valores inferiores, também foi verificado em bulbos de S. multiflorus (Amaryllidaceae) e rizomas de P. nivalis (Orchidaceae). Para essas duas espécies o conteúdo de sacarose foi de $20 \%$ da MS e $65 \%$, respectivamente. A maior concentração de frutose na fração de oligossacarídeos (etanólica) se deve às propriedades físico-químicas dessa cetose que possui maior solubilidade em solução etanólica aquecida (Pollock \& Jones 1979).

Bulbos com elevados teores de carboidratos nãoestruturais é característica bastante citada na literatura. Stancato et al. (1995) relataram elevados teores de CST em bulbos da ornamental Hippeastrum hybridums (Amaryllidaceae) em que $58 \%$ da MS de seus bulbos era constituída de sacarose e $22 \%$ de amido. Em relação a essa espécie, os teores de sacarose em $H$. reticulatum da restinga do Espírito Santo são similares enquanto o teor de amido foi muito baixo, correspondendo apenas a $2 \%$ da MS. Shin et al. (2002) verificaram, também, elevados teores de sacarose em bulbos de Lilium (Liliaceae).

Além de ser amplamente utilizada como adoçante natural, a sacarose é empregada, também, na indústria para a produção de álcool combustível. A sacarose tem grande importância ecológica para as espécies vegetais que vivem em ambientes que passam por algum tipo de estresse ambiental. A resistência de algumas plantas ao déficit hídrico parece estar relacionada ao acúmulo de carboidratos solúveis de baixa massa molecular que podem reduzir o potencial osmótico (Chaves Filho \& Stacciarini-Seraphin 2001, Stancato et al. 2001). O teor elevado de sacarose encontrado em $P$. nivalis e sua distribuição geográfica no Brasil, restrita a ambientes rupestres (Pabst \& Dungs 1977), permite associar a sacarose dessa Orchidaceae com os mecanismos de resistência ao estresse hídrico temporário. Além dos carboidratos, as orquídeas possuem atributos morfoanatômicos e mecanismos fisiológicos eficientes como o metabolismo ácido de Crassuláceas que auxiliam na economia de água nos períodos de maior deficiência hídrica (Braga 1977; Oliveira \& Sajo 1999).

Os teores consideráveis de frutose encontrados em $H$. reticulatum (20\% da MS) e em $S$. multiflorus, ambas da família Amaryllidaceae, e na Orchidaceae $P$. nivalis ( $8 \%$ de MS) podem ser um indicativo da presença de frutanos uma vez que esses polímeros de frutose são encontrados abundantemente em órgãos subterrâneos em Amaryllidaceae de regiões temperadas e em Liliaceae (Vijn \& Smeekens 1999). Para a confirmação dessa hipótese faz-se necessário uma análise mais detalhada dos seus carboidratos por cromatografia líquida de alta eficiência (CLAE), uma vez que nenhuma referência foi encontrada a respeito da composição de carboidratos em $H$. reticulatum, $S$. multiforus e $P$. nivalis. A busca de novas culturas produtoras de frutanos é grande. Embora as maiores fontes comerciais de frutanos são raízes de Dhalia sp., Helianthus tuberosus e Cichorium intybus de regiões temperadas (Cuzzuol et al. 2003), a descoberta de novas plantas tropicais produtoras desses carboidratos é de grande interesse para a indústria alimentícia e farmacêutica. No cerrado, mais de $50 \%$ das espécies da família Asteraceae produzem frutanos do tipo inulina em seus órgãos subterrâneos, especialmente em Vernonia herbacea (Tertuliano \& Figueiredo-Ribeiro 1993) em outras espécies de dicotiledôneas tais como Gomphrena macrocephala (Amaranthaceae) que acumulam frutanos do tipo fleanos em suas raízes tuberosas (Vieira \& Figueiredo-Ribeiro 1993). Os benefícios dos frutanos à saúde humana são enormes. São utilizados como probióticos das bifidobactérias intestinais controladoras da incidência de doenças intestinais como câncer de cólon e estimulantes da absorção de cálcio, magnésio e ferro, promovendo a densidade mineral óssea e recuperação das hemácias nos casos de anemia (Coussement \& Franck 1998). A sua elevada capacidade higroscópica, formando estruturas semelhantes a géis, faz desse ingrediente alimentar um excelente espessante de alimentos, permitindo o seu uso como substituto de gorduras e estabilizante de alimentos (Silva 1996).

Quando comparada a Sinningia allagophylla, uma Gesneriaceae do cerrado, cujo conteúdo de amido corresponde a $50 \%$ da MS (Almeida 1994), os resultados obtidos com S. aghensis da flora do Espírito Santo parecem contraditórios uma vez que o amido representou apenas $1 \%$ de sua MS. No entanto, Müller et al. (1997), trabalhando com outros gêneros da família Gesneriaceae, constataram resultados similares ao verificado em S. aghensis, em que a sacarose foi o carboidrato solúvel mais representativo e o amido, o menos expressivo. A distribuição dessa Gesneriaceae restrita a ambientes rupestres do ES, têm levado alguns autores a classificar outras espécies dessa família como revivescente devido a presença da sacarose, metabólito chave nos mecanismos de osmoregulação em plantas que passam por restrição hídrica (Müller et al. 1997). 
Os tubérculos das espécies do gênero Dioscorea sp. 1 (Dioscoreacee) estudados no presente trabalho apresentaram elevado conteúdo de amido (50\% da MS). Tratando-se de alimentação humana as espécies do gênero Dioscorea possuem tubérculos ricos em amido contendo vitaminas do complexo B, sendo conhecidos no Brasil como cará. Algumas espécies de Dioscoreaceae podem apresentar propriedades físico-químicas apropriadas para a dieta alimentar (Leonel \& Cereda 2002). O amido difere dos demais carboidratos por ser um polímero de glicose, complexo e insolúvel em água. Segundo Alves \& Grossmann (2002), a quantidade elevada de amido em Dioscorea alata (inhame) demonstra ser viável seu uso na indústria alimentícia para fabricação de biscoitos. De acordo com Leonel \& Cereda (2002), embora o gênero Dioscorea seja rico em amido com potencial de produção estimado em 6,1 toneladas por hectare, industrialmente ele é menos utilizado que o amido de mandioca. Esse fato deve-se a grande quantidade de mucilagens que mantêm o amido em suspensão dificultando sua sedimentação.

As indústrias alimentícias são as maiores consumidoras de amido. Entretanto, este polissacarídeo é usado também em um grande número de processos industriais destacando-se seu uso pelas indústrias química e têxtil. Atualmente, devido às restrições aos amidos modificados impostas principalmente pelas indústrias alimentícias (Messenger 1997; Leonel \& Cereda 2002), as empresas produtoras de amido vêm demonstrando um interesse crescente em amidos naturais com características que atendam ao mercado consumidor. Desse modo, embora o conteúdo de amido em Dioscorea sp. 1 (50\% da MS) e Dioscorea sp. 2 (13\% da MS) sejam diferentes entre si e de outras plantas produtoras de amido, análises químicas mais refinadas poderiam revelar propriedades físico-químicas de grande interesse para o mercado consumidor de Dioscoriaceae ocorrente na flora do Espírito Santo.

Além do potencial das plantas nativas como produtoras de carboidratos com funções fisiológicas, uma outra importância econômica de plantas portadoras de órgãos de reserva subterrâneos consiste na sua utilização como ornamentais (Fig. 1). O gênero Hippeastrum contém várias espécies de valor ornamental. São conhecidas como amarílis e algumas delas, devido à combinação de vermelho e verde-escuro, são favoráveis para comercialização no natal e ano novo, uma vez que seu florescimento pode ser manipulado para esses períodos (Silberbush et al. 2003). Outras espécies com características ornamentais são Hedychium coronarium, uma macrófita aquática, exótica e invasora nativa do Himalaia, popularmente conhecida como líriodo-brejo ou mariazinha-do-brejo, e Sinningia aghensis, uma gloxínia rupestre de grande beleza ornamental.
Além dos órgãos subterrâneos, as sementes são outras importantes estruturas de armazenamento de compostos nutritivos sendo os galactomanano, o galactano e o xiloglucano os polissacarídeos de parede celular mais abundantes em sementes (Buckeridge et al. 2004). O consumo dessas fibras proporciona uma série de benefícios ao organismo humano tendo sido relatados resultados satisfatórios na prevenção de algumas doenças crônicas. Melhoria das funções gastrointestinais, redução dos níveis de colesterol e glicemia são alguns dos efeitos fisiológicos promovidos pelas fibras biológicas (López et al. 1997). A aplicação de PRPC na dieta humana já é uma realidade. Farinha da semente do jatobá-do-cerrado possui conteúdo elevado de fibra alimentar com grande potencial para utilização na preparação de produtos alimentícios (Silva et al. 1999).

Das espécies analisadas no presente trabalho, H. courbaril mostrou maiores teores de PRPC chegando a representar $70 \%$ da massa seca de suas sementes. Esse maior valor em relação àquele encontrado em jatobá da flora de São Paulo (40\% de xiloglucano) por Buckeridge \& Dietrich (1990) denota diferenças quantitativas e, possivelmente, até qualitativas do xiloglucano extraído em sementes de Jatobá da Mata Atlântica do Espírito Santo. Tal comportamento é relatado para $H$. courbaril de diferentes localidades do território brasileiro (Buckeridge \& Dietrich 1990; Freitas et al. 2005) e para Copaifera langsdorfii cujo conteúdo de polissacarídeos foi maior em indivíduos de Mata Atlântica do que aqueles procedentes do cerrado paulista (Buckeridge et al. 1992).

$\mathrm{Em} C$. grandis foram encontrados elevados teores de polissacarídeos (53\% da MS) em suas sementes. A presença de polissacarídeos do tipo galactomanano em espécies do gênero Cassia como C. grandis $(50 \%$,) (Kapoor \& Joshi 2003), C. spectabilis DC (40\%) (Kapoor et al. 1998) e C. angustifolia Vahl (Chaubey \& Kapoor 2001) nas florestas tropicais da Índia sugere que os polissacarídeos de $C$. grandis e $C$. fistula ocorrentes na flora do ES sejam também do tipo galactomanano. Dada a elevada concentração de polissacarídeos em $C$. grandis, essa espécie poderia ser recomendada como fonte de galactomananos com propriedades físico-químicas semelhantes ao de $H$. courbaril.

Depois de $H$. courbaril e $C$. grandis, $C$. rosea foi a espécie que apresentou maior concentração de PRPC (40\% da MS), porém inferior ao verificado na arbórea Canavalia ensiformes, Canavalia marítima e Canavalia obtusifolia (Sridhar \& Seena 2006). A identificação de polissacarídeos do tipo galactomananos em Canavalia ensiformis (Oliveira et al. 1999b), sugere que o polissacarídeo encontrado em $C$. rosea da restinga do Espírito Santo também seja do tipo galactomanano 
por serem espécies do mesmo gênero. Uma das propriedades desse composto está relacionado aos mecanismos de defesa das plantas. Canavalia ensiformes possui diversos metabólitos antinutricionais como a lectina, canavalina A, uréases, inibidores de proteases e canatoxina que em conjugação com os galactomananos têm efeito tóxico no desenvolvimento das larvas de insetos (Oliveira et al. 1999a; b). A ausência de sementes com sinais de herbivoria (dados não mostrados) em $C$. rosea pode indicar a presença de compostos antinutricionais com função antiherbivoria como constatado em Canavalia ensiformes (Oliveira et al. 1999a; b) e em outras espécies do Gênero Canavalia (Sridhar \& Seena 2006).

A concentração de polissacarídeos em sementes de E. variegata foi muito baixa (Fig. 5) representando apenas $10 \%$ de sua MS, sendo esse valor menor ainda em $O$. arborea (3\%) denotando que essas duas espécies possuem baixo potencial para exploração de polissacarídeos funcionais. A inexistência de referências bibliográficas a respeito da constituição química dos polissacarídeos dos gêneros Erytrina e Ormosia não permite hipotetizar a respeito da estrutura química dos PRPC de E. variegata e O. arborea.

Semelhante a essas duas espécies, a análise de compostos de reserva em sementes de plantas nativas não é muito explorado apesar da grande diversidade e biomassa vegetal existente em florestas tropicais brasileiras.

Com relação aos nutrientes minerais, os elevados teores de $\mathrm{N}$ em $E$. variegata e $C$. rosea indicam elevado conteúdo de proteínas podendo representar até $25 \%$ da massa seca em Canavalia ensiformes (Agbede et al. 2003) e até $39 \%$ em outras espécies do gênero Canavalia (Sridhar \& Seena 2006), porém com valores inferiores aos encontrados em leguminosas cultivadas. Os teores expressivos de $\mathrm{K}, \mathrm{Ca}, \mathrm{Pe} \mathrm{Mg} \mathrm{em} \mathrm{E}$. variegata e $C$. rosea embora sejam relativamente inferiores à soja, são superiores a de outras leguminosas (Oke et al. 1995) indicando a potencialidade dessas espécies como alternativa terapêutica em tratamentos para estimulação da mineralização óssea e balanço osmótico. Os elevados teores de Fe e $\mathrm{Zn}$ em E. variegata e C. rosea mostra, mais uma vez, o potencial nutritivo das sementes dessas espécies com função fisiológica para tratamento de anemia e controle de infecções causadas pela deficiência $\mathrm{Zn}$, importante estimulador do sistema imunológico (Cook et al. 2000). Dessa forma, a utilização de farinha dessas sementes, após testes toxicológicos, poderia ser utilizada como suplemento nutritivo na dieta alimentar de populações com problemas de desnutrição.

Como constatado no presente trabalho, os estudos relacionados à bioprospecção revelam resultados promissores mostrando o grande potencial das plantas ocorrentes na flora do Espírito Santo para produção de carboidratos com função fisiológica e suplemento nutricional. Ressaltamos que grande parte dos alimentos originou-se de regiões tropicais, onde se encontram os parentes selvagens das espécies cultivadas. Tais indivíduos possuem materiais genéticos que podem ser usados para modificar o cultivo atual e produzir variedades resistentes a pestes e doenças. Essa imensa riqueza natural constitui-se num verdadeiro patrimônio cientifico e cultural que precisa ser conhecido, preservado e explorado. Além disso, trabalhos dessa natureza permitirão fornecer subsídios para programas de desenvolvimento sustentável e, ainda, estabelecer estratégias de conservação da flora nativa das florestas tropicais do Espírito Santo.

\section{Agradecimentos}

À CAPES, pela concessão de bolsa de mestrado à primeira autora; à Profa. Dra. Lílian Beatriz Penteado Zaidan e Profa. Dra. Luciana Dias Thomaz, pela revisão e sugestões.

\section{Referências bibliográficas}

Agbede, J.O. \& Aletor, V.A. 2003. Studies of the chemical composition and protein quality evaluation of differently processed Canavalia ensiformis and Mucuna pruriens seed flours. Journal of Food Composition and Analysis 18: 89-103.

Almeida, V.P. 1994. Tuberização em Sinningia allagophylla (Martius) Wiehler (Gesnereaceae), uma espécie do cerrado. Tese de Doutorado. Campinas, SP, Universidade Estadual de Campinas.

Alves, R.M.L. \& Grossmann, M.V.E. 2002. Yam flour for expanded snacks. Ciência Tecnologia Alimentícia 22: 32-38.

Bataglia, O.C.; Furlani, A.M.C.; Teixieira, J.P.F.; Furlani, P.R. \& Gallo, J.R. 1983. Métodos de análise química de plantas. Boletim Técnico 78. Instituto Agronômico de Campinas.

Braga, P.I.S. 1977. Aspectos ecofisiológicos de Orchidaceae da Amazonia II. Anatomia ecológica foliar de espécies com metabolismo CAM de uma campina da amazonia central. Acta Amazonica 7: 1-89.

Bello, J. 1995. Los alimentos funcionales nutraceuticos: funciones saludables de algunos componentes de los alimentos. Alimentaria 267: 49-58.

Buckeridge, M.S. \& Dietrich, S.M.C. 1990. Galactomannan from Brazilian legume seeds. Revista Brasileira de Botânica 13: 109-112.

Buckeridge, M.S.; Rocha, D.C.; Reid, J.S.G. \& Dietrich, S.M.C. 1992. Xyloglucan structure and post-germinative metabolism in seeds of Copaifera langsdorfii from savana and forest populations. Physiology Plantarum 86: 145-151.

Buckeridge, M.S.; Aidar, M.P.M.; Santos, H.P. \& Tiné, M.A. 2004. Acúmulo de Reservas. Pp. 31-50. In: A.G. Ferreira \& F. Borgheti (eds.). Germinação: do básico ao aplicado. Porto Alegre, Artmed.

Carvalho, M.A.M. \& Dietrich, S.M.C. 1993. Variation in fructan content in the undrground organs of Vernonia herbacea (Vell.) Rusby at different phenological phases. New Phytologist 123: 735-740. 
Carvalho, M.A.M.; Pinto, M.M. \& Figueiredo-Ribeiro, R.C.L. 1998. Inulin production by Vernonia herbacea as influenced by mineral fertilization and time of harvest. Revista Brasileira de Botânica 21: $275-280$

Carvalho, M.A.M. \& Figueiredo-Ribeiro, R.C.L. 2001. Frutanos ocorrência, estrutura e composição, com ênfase em plantas do cerrado. Pp.77-89. In: F.M. Lajolo; F. Saura-Calixto; E.W. Penna \& E.W. Menezes (eds.). Fibra Dietética en Iberoamerica: Tecnologia y Salud. Varela Editora e Livraria Ltda.

Chaubey, M. \& Kapoor, V.P. 2001. Structure of a galactomannan from the seeds of Cassia angustifolia Vahl. Carbohydrate Research 332: 439-444.

Chaves Filho, J.T. \& Stacciarini-Seraphin, E. 2001. Alteração no potencial osmótico e teor de carboidratos solúveis em plantas jovens de lobeira (Solanum lycocarpum St.-Hil.) em resposta ao estresse hídrico. Revista Brasileira de Botânica 24: 199-204.

Cook, J.A.; Vanderjagdt, D.J.; Pastuszyn, A.; Mounkaila, G.; Glew, R.S.; Millson, M. \& Glew, R.H. 2000. Nutrient and Chemical Composition of 13 Wild Plant foods of Niger. Journal of Food Composition and Analysis 13: 83-92.

Coussement, P. \& Franck, A. 1998. New food applications for inulin. Agro food Industry Hi-Tech 9: 26-28.

Crepaldi, I.A.; Almeida-Muradian, L.B.; Rios, M.D.G.; Penteado, M.V.C. \& Salatino, A. 2001. Composição nutricional do fruto de licuri (Syagrus coronata (Martius) Beccari). Revista Brasileira de Botânica 24: 155-159.

Cuzzuol, G.R.F.; Carvalho, M.A.M.; Barbedo, C.J. \& Zaindan, L.B.P. 2003. Crescimento e conteúdo de frutanos em plantas de Vernonia herbacea (Vell.) Rusby submetidas à adubação nitrogenada. Revista Brasileira de Botânica 26: 81-91.

Cuzzuol, G.R.F.; Carvalho, M.A.M. \& Zaidan, L.B.P. 2005. Growth, photosynthate partitioning and fructan accumulation in plants of Vernonia herbacea (Vell.) Rusby under two nitrogen levels. Brazilian Journal of Plant Physiology 17: 401-410.

Dennis, D.T. \& Blakeley, S.D. 2000. Carbohydrate metabolism. In: B.B. Buchanan; W. Gruissem \& R.L. Jones (eds.). Biochemistry \& Molecular Biology of Plants. Rockville, American Society of Plant Physiologists.

Dias-Tagliacozzo, G.M.; Itaya, N.M.; Carvalho, M.A.M.; FigueiredoRibeiro, R.C.L. \& Dietrich, S.M.C. 2004. Fructans and water suppression on intact and fragmented rhizophores of Vernonia herbacea. Brazilian Archives of Biology and Technology 47: $363-373$

Dubois, M.; Gilles, K.A.; Hamilton, J.K.; Rebers, P.A. \& Smith, F. 1956. Colorimetric method for the determination of sugars and related substances. Analytical Chemistry 28: 350-356.

EMBRAPA. Centro Nacional de Pesquisa de Solos. 1997. Manual de métodos de análise de solo. EMBRAPA-CNPS

Flores, H.E. \& Flores, T. 1997. Biology and biochemistry of underground plant storage organs. Pp. 113-132. In: T. Johns \& H. Romeo (eds.). Functionality of Food Phytochemicals. New York, Plenum Press.

Freitas, R.A.; Martin, S.; Santos, G.L.; Valenga, F.; Buckeridge, M.S.; Reicher, F. \& Sierakowski, M.R. 2005. Physico-chemical properties of seed xyloglucans from different sources. Carbohydrates Polymers 60: 507-514.

Gonçalves, J.F.C.; Fernandes, A.V. \& Oliveira, A.F.M. 2002. Primary metabolism components of seeds from Brazilian Amazon tree species. Brazilian Journal of Plant Physiology 14: 139-142.

Jermyn, M.A. 1956. A new method for determination of ketohexoses in presence of aldohexoses. Nature 177: 38-39.

Kapoor, V.P. \& Joshi, H. 2003. Cassia grandis Linn. f. seed galactomannan: structural and crystallographical studies. Carbohydrate Research 338: 1907-1912.

Kapoor, V.P.; Taravel, F.R.; Joseleau, J.P.; Milas, M.; Chanzy, H. \& Rinaudo, M. 1998. Cassia spectabilis DC seed galactomannan: structural, crystallographical and rheological studies. Carbohydrate Research 306: 231-241.
Leonel, M. \& Cereda, M.P. 2002. Caracterização físico-química de algumas tuberosas amiláceas. Ciência Tecnologia Alimentícia 22: 65-69.

López, G.; Ros, G.; Rincón, F.; Periago, M.J.; Martínez, C. \& Ortuno, J. 1997. Propriedades funcionales de la fibra dietética. Mecanismos de acción em el tracto gastrointestinal. Archivos Latinoamericanos de Nutrición 47: 203-207.

McCready, R.M.; Guggolz, J.; Silveira, V. \& Owens, H.S. 1950. Determination of starch and amylose in vegetables: application to peas. Analytical Chemistry 22: 1156-1158.

Messenger, B. Going native. 1997. Food processing 1: 58-59

Morais, G.A. 2000. Germinação e Desenvolvimento inicial de Hymenaea courbaril L.: efeito de condições ambientais contrastantes e da remoção cotiledonar. Tese de Doutorado. Rio Claro, Universidade Estadual Paulista.

Müller, J.; Sprenger, N.; Bortilik, K.; Boller, T. \& Wiemken, A. 1997. Desiccation increases sucrose levels in Ramonda and Haberlea, two genera of resurrection plants in the Gesneriaceae. Physiology Plantarum 100: 153-158

Oke, D.B.; Tewe, O.O. \& Fetuga, B.L. 1995. The nutrient composition of some cowpea varieties. Nigerian Journal of Animal Production 22: 32-36

Oliveira, A.E.A.; Gomes, V.M.; Sales, M.P.; Fernandes, K.V.S.; Carlini, C.R. \& Xavier Filho, J. 1999a. The toxicity of Jack bean (Canavalia ensiformis) L. DC. canatoxin to plant pathogenic fungi. Revista Brasileira de Biologia 59: 59-62.

Oliveira, A.E.A.; Sales, M.P.; Machado, O.L.T.; Fernandes, K.V.S. \& Xavier Filho, J. 1999b. The toxicity of Jack bean (Canavalia ensiformis) cotyledon and seed coat proteins to the cowpea weevil (Callosobruchus maculatus). Entomologia Experimentalis et Applicata 92: 249-255.

Oliveira, V.D.C. \& Sajo, M.D.G. 1999. Anatomia foliar de espécies epífitas de Orchidaceae. Revista Brasileira de Botânica 22: 365-374.

Pollock, C.J. \& Jones, T. 1979. Seasonal patterns of fructan and metabolism in forage grasses. New Phytologist 83: 8-15.

Pabst, G.F.J. \& Dungs, F. 1977. Orchidaceae Brasilienses. BruckVerlag.

Riazi, A. Motsuda, K. \& Arslon, A. 1985. Water - Stress induced changes in concentrations of prolines and other solutes in growes regions of young barley leaves. Journal of Experimental Botany 172: 1716-1725.

Saeman, J.F.; Buhl, J.L. \& Harris, E.E. 1945. Quantitative saccharification of wood and cellulose. Production of Wood Sugar in Germany and Its Conversion to Yeast and Alcohol 17: 35-37.

Shin, K.S.; Chakrabarty, D. \& Paek, K.Y. 2002. Sprouting rate change of carbohydrate contents and related enzymes during cold treatment of lily bulblets regenerated in vitro. Scientia Horticulturae 96: 195-204.

Silberbush, M.; Ephrath, J.E.; Alekperov, C.E. \& Ben-Asher, J. 2003. Nitrogen and potassium fertilization interactions with carbon dioxide enrichment in Hippeastrum bulb growth. Scientia Horticulturae 98: 85-90.

Silva, R.F. 1996. Use of inulin as a natural texture modifier. Ceral Foods World 41: 792-794

Silva, M.R.; Silva, M.A.A.P. \& Chang, Y.K. 1999. Uso de farinha de jatobá (Hymenaea stigonocarpa Mart.) em biscoitos tipo "cookie". Alimentação e Nutrição 10: 7-22.

Silva, C.O.; Minhoto. M.J. \& Buckerdige, M.S. 2005. Biodiversidade, mudanças climáticas e a tabela brasileira de composição de alimentos. Pp. 29-62. In: E. Salay. (org.). Composição de alimentos: uma abordagem multidisciplinar. NEPA/UNICAMP.

Sridhar, K.R. \& Seena, S. 2006. Nutritional and antinutritional significance of four unconventional legumes of the genus Canavalia - A comparative study. Food Chemistry 99: 267-288.

Stancato, G.C.; Mazzafera, P. \& Magalhães, A.C.N. 1995. Dry matter partitioning during the propagation of Hippeastrum hybridum as affected by light. Scientia Agricola 62: 81-87. 
Stancato, G.C.; Mazzafera, P. \& Buckeridge, M.S. 2001. Effect of a drought period on the mobilization on non-structural carbohydrates, photosynthetic efficiency and water status in an epiphytic orchid. Plat Physiology and Biochemistry 39: 1009-1016.

Tertuliano, M.F. \& Figueiredo-Ribeiro, R.C.L. 1993. Distribution of fructose polymers in herbaceous species of Asteraceae from the cerrado. New Phytologist 123: 741-749.

Thomaz, L.D. \& Monteiro, R. 1993. Distribuição das espécies na comunidade halófila-psamófila ao longo do litoral do Espírito Santo. Arquivos de Biologia e Tecnologia 36: 375-399.
Thomaz, L.D. \& Monteiro, R. 1997. Composição Florística da Mata Atlântica de encosta da Estação Biológica de Santa Lúcia, município de Santa Teresa-ES. Boletim do Museu de Biologia Mello Leitão 7: 3-48.

Vieira, D.J. \& Figueiredo-Ribeiro, R.C.L. 1993. Fructose-containing carbohydrates in the tuberous root of Gomphrena macrocephala St.-Hil. (Amaranthaceae) and different phenological phases. Plant, Cell and Environment 16: 919-928.

Vijn, I. \& Smeekens, S. 1999. Fructan: more than a reserve carbohydrate? Plant Physiology 120: 351-359.

Yexkull, H.R. \& Mutert, E. 1995. Global extent, development and economic impact of acid soils. Plant Soil 171: 1-15.

Versão eletrônica do artigo em www.scielo.br/abb e http://www.botanica.org.br/acta/ojs 